\title{
Response to Letter-to-the-Editor Surgical Care: Addressing the Barriers to Access in Low-Income Countries
}

\author{
Lubna Samad · Fayez Jawed • Naila Baig-Ansari
}

Published online: 5 December 2013

(C) Société Internationale de Chirurgie 2013

We appreciate the interest shown by Ologunde and Holmer in our findings. We agree that delays can occur at several points from the time of onset to the time of resolution of symptoms. The "three delays" framework defined for emergency obstetrical care [1] highlights the all too common situation where a health facility is unable to provide the required care. A long, expensive journey to a hospital where adequate personnel, resources, or expertise are not available discourages other people in the community from seeking care in similar situations. The delay defined by Ologunde and Homer is one that often is overlooked, largely because surgeons review only those patients who follow-up as scheduled during the postoperative phase. It is usually beyond the capacity of most facilities to track patients who do not show up in clinic; this may be for a variety of reasons, ranging from dissatisfaction with the care received to social and financial barriers. Most tertiary care services, especially in the developing world, are standalone facilities that do not function as part of an integrated health network, where the responsibility for continuity of care is shared between general practitioners and health facilities, providing a safety net for the patient. The continuing education and involvement of general practitioners would help to strengthen the overall continuum of health care.

\footnotetext{
L. Samad $(\bowtie) \cdot$ F. Jawed $\cdot$ N. Baig-Ansari

Indus Hospital Research Center, 4th floor, Indus Hospital,

Korangi Crossing, Karachi, Pakistan

e-mail: lubna.samad@irdresearch.org

F. Jawed

e-mail: fayez.jawed@irdresearch.org

N. Baig-Ansari

e-mail: naila.baigansari@irdresearch.org
}

We agree that long-standing lack of access has led to patients becoming less inclined to seeking care. In addition to strengthening primary care networks, secondary and tertiary level facilities that provide sustained high-quality surgical care that is affordable and accessible for the community need to exist. Our facility provides free of cost care of a high standard in a low income, underserved population of Karachi. Within a short span of 7 years, the hospital services have been overwhelmed to the extent that an expansion to increase capacity by more than tenfold is currently underway.

The parameters used to assess the success of surgery mainly center on immediate postoperative morbidity and mortality rates, as well as adverse events. In some cases, our long-term concern extends to the survival rate of our cancer patients. Very little emphasis is placed on a patient's long-term quality of life (QoL) after surgery; this QoL assessment gap is reflected in our research interests as well. Hardly any research exists from developing countries in assessment of QoL, which includes pain assessment or functionality following surgery-all areas that would influence care seeking behaviour in other patients [2]. We agree with Ologunde and Homer's view on this matter and emphasize the need for systematic assessments on how best to redress this gap.

\section{References}

1. Barnes-Josiah D, Myntti C, Augustin A (1998) The "three delays" as a framework for examining maternal mortality in Haiti. Soc Sci Med 46:981-993

2. Fraser SC (1993) Quality-of-life measurement in surgical practice. Br J Surg 80:163-169 